Research Article

\title{
Transcriptional activity of the 5'-flanking region of the thyroid transcription factor-1 gene in human thyroid cell lines
}

Hiroya Yamada ${ }^{1,2}$, Toru Takano ${ }^{1}$, Fumio Matsuzuka ${ }^{3}$, Mikio Watanabe ${ }^{2}$, Akira Miyauchi $^{3}$ and Yoshinori Iwatani ${ }^{2}$

${ }^{1}$ Department of Laboratory Medicine, Osaka University Graduate School of Medicine, Suita, Osaka, Japan.

${ }^{2}$ Division of Health Sciences, Osaka University Graduate School of Medicine, Suita, Osaka, Japan.

${ }^{3}$ Kuma Hospital, Chuo-ku, Kobe, Hyogo, Japan.

\begin{abstract}
Thyroid transcription factor-1 (TTF-1, NKX2-1) is a homeodomain-containing transcriptional factor that binds to and activates the promoters of thyroid and lung-specific genes, such as thyroglobulin, thyroid peroxidase, and thyroid stimulating hormone receptor. TTF-1 is known to play a key role in the development of the thyroid. However, the precise mechanism of TTF-1 gene transcription in human thyroid cells has not been studied. The expression of transcriptional activity in various lengths of the 5'-flanking region of the human TTF -1 gene was studied in TTF-1 positive and negative human thyroid cell lines. Increased transcriptional activity was observed in thyroid cell lines containing plasmids that coded for a sequence proximal to the transcription start site of exon 1 of the TTF-1 gene. However, we did not observe any difference in promoter activity in the region up to $-2.6 \mathrm{~kb}$ from the proximal transcription start site of the TTF-1 gene between TTF-1 positive and negative cells. These results suggest that the proximal 5'-flanking region of the human TTF -1 gene does not contain sufficient cis-active regulatory information to direct gene expression in thyroid cells, and that other cis- or trans-acting factors participate in the thyroid specific gene expression of TTF-1.
\end{abstract}

Key words: thyroid, TTF-1, transcription, human cell line, stem cell.

Received: May 12, 2010; Accepted: July 29, 2010.

\section{Introduction}

Thyroid transcription factor-1 (TTF-1, NKX2-1) is a homeodomain-containing transcriptional factor that binds to and activates the promoters of thyroid and lung epithelial genes. TTF-1 expression becomes detectable in the thyroid primordium as it begins to migrate caudally from just above the aortic sac in E9-E10 embryos (Lazzaro et al., 1991). Thus, the expression of TTF-1 is regarded as a potentially useful marker for selecting and identifying fetal thyroid cells in studies analyzing the regeneration of thyroid follicular cells or thyroid cancer stem cells (Takano, 2007; Klonisch et al., 2009). Furthermore, it is necessary for the activation of thyroid specific genes, such as thyroglobulin, thyroid peroxidase, and thyroid-stimulating hormone receptor. The absence of wild type TTF-1 activity results in extensive phenotypic and functional abnormalities in the thyroid (Vliet, 2003).

In cell lines derived from the lung, a proximal 5'flanking region of the TTF-1 gene of $2.7 \mathrm{~kb}$ in length has

Send correspondence to Toru Takano. Department of Laboratory Medicine, Osaka University Graduate School of Medicine, D2 2-2 Yamadaoka, Suita, 565-0871 Osaka, Japan. E-mail: ttakano@labo.med.osaka-u.ac.jp. been reported to show strong transcriptional activity (Ikeda et al., 1995). Even though TTF-1 plays a key role in early thyroid development, the precise mechanism of TTF-1 gene transcription in thyroid cells remains mostly unknown. Pan et al. (2004) introduced DNA from the TTF-1 5' DNA flanking region to induce direct expression of LacZ in transgenic mice during embryonic development. These transgenic mice expressed LacZ in the lung but not in the thyroid. These results indicate that, at least in mice, the regulatory elements that are essential for TTF-1 expression in thyroid cells are located in the genomic regions outside of the TTF-1 5'-flanking region. Nakazato et al. (1997) analyzed the transcriptional activity of the rat TTF-1 gene in the FRTL-5 rat thyroid cell line. They observed strong transcriptional activity in the 5'-flanking region from -2507 to -1892 but not in the region from -2021 to -1892 and concluded that the sequence from -2507 to -2021 of the rat TTF-1 gene exhibits tissue-selective promoter activity. Furthermore, Oguchi and Kimura (1998) transfected rat TTF-1 gene sequences of various lengths into FRTL-5 cells and concluded that a $-1.94 \mathrm{~kb}$ upstream sequence is required for maximal promoter activity. The results in these three studies differed, and no study using human thyroid 
cells has been published, probably because until recently there were no known human thyroid cell lines that were able to abundantly express TTF-1.

In a recent study we found two anaplastic thyroid carcinoma (ATC)-derived cell lines, TCO-1 and 8505C, that expressed TTF-1 mRNA (Takano et al., 2007). Thus, in the present paper, we investigated TTF-1 5'-flanking region transcriptional activity in TCO- 1 and $8505 \mathrm{C}$ cells and compared the results with those for other ATC cell lines, viz. 8305C and SW571, which do not express TTF-1 mRNA.

\section{Materials and Methods}

Cloning of the 5 '-flanking region of the human TTF-1 gene and construction of TTF-1 reporter plasmid

A 5'-upstream region of the TTF-1 gene was amplified from human genomic DNA using the polymerase chain reaction (PCR), as described previously (Takano et al., 2008). The PCR products were sub-cloned into a pGL4 vector (Promega, Tokyo, Japan) containing a luciferase reporter gene. According to previous studies, the position of the first ATG in the second exon was designated as zero (Oguchi and Kimura, 1998). The human TTF-1 gene has two major transcription start sites. One is upstream of the first exon, and the other is localized in the first intron (Hamdan et al., 1998). The DNA fragments were designed to cover these regions. The cloned fragments TA1-5 and TB2-5 covered the following regions: -210 to $-1,-552$ to $-1,-1272$ to $-1,-2110$ to -1 , and -2744 to -1 and -522 to $-179,-1272$ to $-179,-2110$ to -179 , and -2744 to -179 , respectively.

\section{Cell culture}

Four cell lines derived from human ATC namely TCO-1, 8505C, SW571, and 8305C, were cultured in RPML-1640 with 10\% fetal bovine serum (Invitrogen, Tokyo, Japan) at $37{ }^{\circ} \mathrm{C}$ in $5 \% \mathrm{CO}_{2}$. HepG2 cells derived from human liver were cultured in DMEM with 10\% fetal bovine serum (Invitrogen) at $37{ }^{\circ} \mathrm{C}$ in $5 \% \mathrm{CO}_{2}$. All cell lines were provided by the Human Science Research Resource Bank (Osaka, Japan).

\section{Transfection and luciferase assay}

$24 \mathrm{~h}$ before transfection, the cells were plated in a 24-well plate at a density of 200,000 cells/well and allowed to grow under normal culture conditions. A promoter-less pGL4 vector was used as control. The plasmid pRL-CMV (Promega) containing the Renilla firefly reporter gene was co-transfected into all cells to normalize the results. $400 \mathrm{ng}$ of pGL4-reporter vector containing one of the fragments of the TTF-1 upstream genomic sequence and $5 \mathrm{ng}$ of the pRL-CMV vector were co-transfected into each cell using $1.2 \mu \mathrm{L}$ of Lipofectamine 2000 (Invitrogen), and the cells were cultured for $48 \mathrm{~h}$. Relative luciferase activity in each cell lysate was measured with the Dual-Luciferase Reporter Assay System (Promega), according to the manufacturer's protocol.

\section{Extraction of RNA from thyroid tissues and cells}

Tissue samples from three normal thyroid tissues from the lobe contralateral to a carcinoma were collected during surgery, after we had obtained informed consent from each patient. The study protocol was approved by the ethics committee of Kuma Hospital. Tissues were frozen in liquid nitrogen immediately after resection. Total RNA of these tissues and cell lines was extracted as described previously (Yamada et al., 2009).

\section{Reverse transcription}

Reverse transcription (RT) was performed using $1 \mu \mathrm{g}$ of total RNA in an RT mixture containing $50 \mathrm{mM}$ Tris- $\mathrm{HCl}$ (pH 8.3), $75 \mathrm{mM} \mathrm{KCl}, 10 \mathrm{mM}$ dithiothreitol, $3 \mathrm{mM} \mathrm{MgCl}_{2}$, $0.5 \mathrm{mM}$ deoxynucleotide triphosphates (dNTP) (Takara, Shiga, Japan), 200 U MMLV reverse transcriptase (Invitrogen), $2 \mathrm{U} / \mu \mathrm{L}$ RNase inhibitor (Takara), and $2.5 \mu \mathrm{M}$ of random hexamers (Takara) in a total volume of $20 \mu \mathrm{L}$ at $42{ }^{\circ} \mathrm{C}$ for $60 \mathrm{~min}$.

\section{Real-time quantitative PCR}

The real-time quantitative PCR using TaqMan probes was performed using an ABI PRISM 7700 Sequence Detection System (Applied Biosystems, Foster City, CA USA), as previously described (Takano et al., 1999) using $1 \mu \mathrm{L}$ of first strand cDNA. The sequence of the two primers and the TaqMan probe used for quantification of TTF-1 mRNA (TITF-1, GenBank BC006221) were:

TTF1F $(0.5 \mu \mathrm{M})$ 5'-TCCAGAACCACCGCTACA3' (bases 746-763),

Table 1 - Summary of the transcriptional activity of the 5' flanking region of the TTF-1 gene described in the present and previous studies.

\begin{tabular}{|c|c|c|c|}
\hline Sequence & Target of transfection & Specific transcriptional activity & Reference \\
\hline Human & Human lung adenocarcinoma cell line & + & Hamdan, Ikeda \\
\hline Human & Mouse lung cell line & + & Ikeda \\
\hline Rat & Rat thyroid cell line & + & Nakazato, Oguchi \\
\hline Mouse & Lung (transgenic mouse) & + & Pan \\
\hline Mouse & Thyroid (transgenic mouse) & - & Pan \\
\hline Human & Human thyroid anaplastic carcinoma cell line & - & Present study \\
\hline
\end{tabular}


TTF1R $(0.5 \mu \mathrm{M})$ 5'- ACGGTTTGCCGTCTTTCA C-3' (bases 928-910), and TTF1-TM (140 nM) 5'-FAM-ACTGCTGCTGA GCCTGTTGCTGC -TAMRA-3' (bases 879-853).

$\beta$-actin (ACTB) mRNA (GenBank X00351) was used as endogenous control.

The sequence of the two primers and of the TaqMan probe used for $A C T B$ mRNA quantification were:

ACF $(0.5 \mu \mathrm{M}) 5^{\prime}$-TGGACATCCGCAAAGACCTG -3' (bases 901-920),

ACR $(0.5 \mu \mathrm{M})$ 5'-CCGATCCACACGGAGTACT T-3' (bases 1066-1047),

and AC-TM (140 nM) 5'-FAM-CACCACCATGTA CCCTGGCATTGCC-TAMRA-3' (bases 947-971).

All primers and probes were purchased from Operon Biotechnologies (Tokyo, Japan). The conditions for TaqMan PCR were: $95{ }^{\circ} \mathrm{C}$ for 10 min and 40 cycles of $95{ }^{\circ} \mathrm{C}$ for $15 \mathrm{~s}$ and $60{ }^{\circ} \mathrm{C}$ for $1 \mathrm{~min}$. Recombinant pGEM T-vectors (Promega, Tokyo, Japan) containing either TTF-1 or $\beta$-actin cDNA were constructed by PCR-cloning, using the same sets of primers as those used for RT-PCR, and then used as standard samples. The amplification plots were used to determine the threshold cycle $\left(\mathrm{C}_{\mathrm{T}}\right)$. The initial mRNA copy number was calculated by plotting $\mathrm{C}_{\mathrm{T}}$ against input target quantity.

\section{Sequence analysis of the 5'-flanking region of the human TTF-1 gene}

Genomic DNA was isolated using a QIAamp DNA Mini Kit (Qiagen, Tokyo, Japan) from five cell lines. The 5'-upstream region of the TTF-1 gene was amplified by PCR, and the PCR products were sub-cloned into a pGEM-Easy $\mathrm{T}$ vector. The cloned fragments covered the region from -2744 to -1 . The purified fragments were sequenced using a BigDye Terminator Cycle Sequencing FS Ready Reaction Kit with an ABI PRISM 310 Genetic Analyzer (Applied Biosystems, Tokyo, Japan). At least three clones were analyzed for each cell line.

\section{Results}

The expression of TTF-1 mRNA was observed in all three normal thyroid tissue samples, as well as in the $8505 \mathrm{C}$ and TCO- 1 cells. In contrast, the $8305 \mathrm{C}$, SW571, and HepG2 cells showed almost no TTF-1 transcripts (Figure 1). When analyzing the sequence of the 5'upstream region of the TTF-1 gene in each cell line, we did not find any mutations or deletions in this region in any of the five cell lines (data not shown).

In the experiments using TA1-5 plasmids, the transcriptional activity of one of the TTF-1 positive cell lines, $8505 \mathrm{C}$, was greatly increased by TA1 compared with the activity observed in the TTF-1 negative cell lines SW571 and $8305 \mathrm{C}$. Nevertheless, another TTF-1 positive cell line, TCO-1, did not demonstrate a clear increase in TTF-1 tran- scription (Figure 2). Thus, there was no plasmid by which TTF-1 positive and negative cell lines would be distinguishable in terms of transcriptional activity. In the thyroid cell lines, a clear increase in transcriptional activity was only observed for TA1-3.

Since TA1, which contained a sequence downstream of the proximal transcription start site, but not TA2 showed extremely high transcriptional activity in $8505 \mathrm{C}$, we constructed another set of plasmids, TB2-TB5, which did not

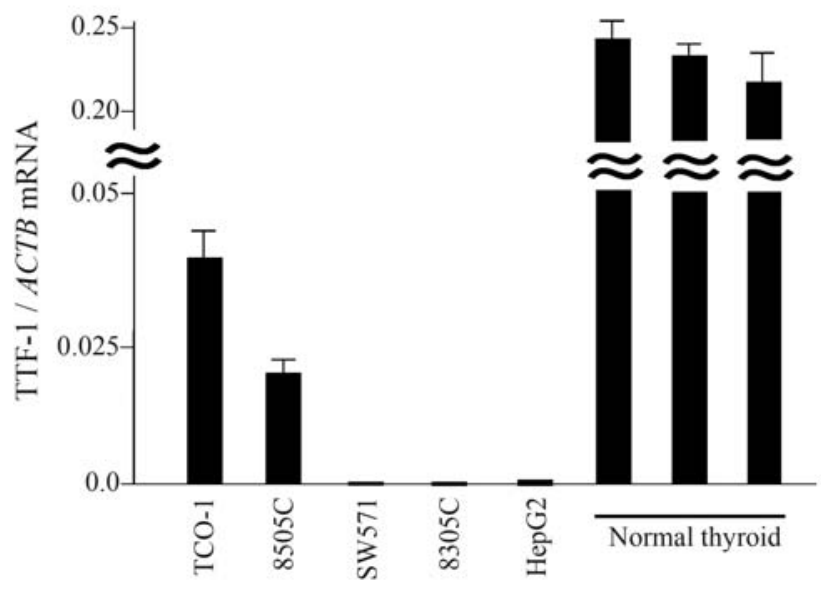

Figure 1 - Expression levels of TTF-1 mRNA relative to $\beta$-actin mRNA. The results are shown as means of duplicate assays.

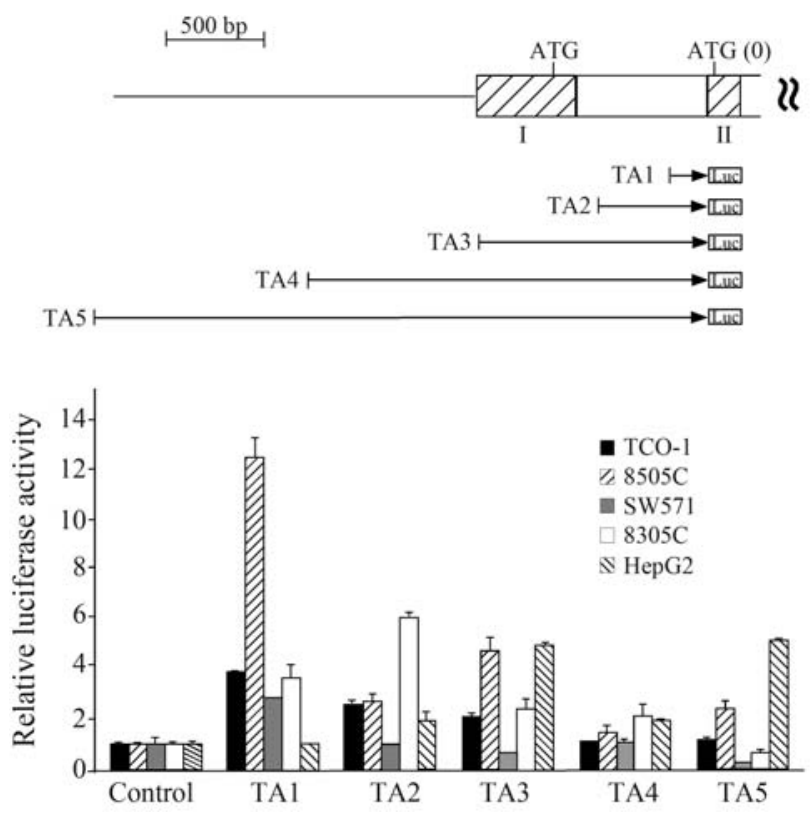

Figure 2 - Schematic diagram of the TTF-1 promoter luciferase constructs and the transcriptional activity of the TTF-1 5' flanking sequences TA1-5. The hatched boxes represent exons while the open boxes represent introns. The base numbers are based on the position of the first ATG in the second exon being 0 . Different lengths of DNA from the 5' flanking region of the human TTF-1 gene were attached to a luciferase reporter plasmid and transfected into five cell lines. Relative luciferase activity obtained with the promoterless pGL4 vector was arbitrarily set as 1 . Values are shown as the mean \pm SD of triplicate assays. 
possess this sequence to test whether this modification would alter the results. When we used these plasmids, a clear decrease in transcriptional activity was observed in $8505 \mathrm{C}$ cells. These results indicated that the high transcriptional activity observed in $8505 \mathrm{C}$ cells following the addition of TA1-5 plasmids was mainly caused by the deleted sequence in exon 2 (Figure 3 ). No plasmid was able to distinguish the TTF-1 positive cell lines from the TTF-1 negative ones in terms of transcriptional activity. Amongst the thyroid cell lines, TB4 and TB5 tended to show weaker transcriptional activity than TB2 and TB3.

\section{Discussion}

This is the first report analyzing the transcriptional activity of the human TTF-1 5'-flanking regions in human thyroid cell lines. The results of previous and the present study on transcriptional activity of the TTF-1 5'- flanking sequence are summarized in Table 1. Although with TA1, one TTF-1 positive cell line, $8505 \mathrm{C}$, showed high transcriptional activity, no increase was observed in another TTF-1 positive cell line, TCO1, or when longer sequences were used, such as TA2-5. Thus, we conclude that no promoter activity was observed that clearly differed between TTF-1 positive and negative cells in the region up to $-2.6 \mathrm{~kb}$ from the proximal transcription start site of the TTF-1 gene. Our results present a clear discrepancy from those of two previous studies using the FRTL-5 rat thyroid cell line, which showed high promoter activity in this region $(\mathrm{Na}-$

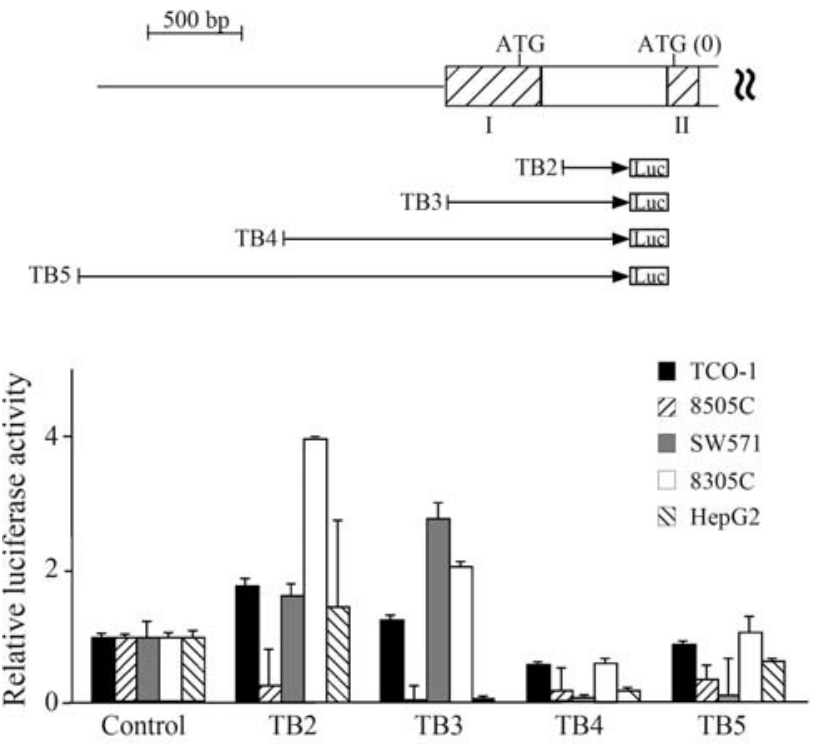

Figure 3 - Schematic diagram of the TTF-1 promoter luciferase constructs and the transcriptional activity of the TTF-1 5' flanking sequences TB2-5. The hatched boxes represent exons while the open boxes represent introns. Base numbers are in relation to the position of the first ATG codon in the second exon, this being set as 0 . The transcription region after the proximal transcription start site has been deleted from TA2-5. The resultant plasmids were transfected into five cell lines. The relative luciferase activity obtained with the promoterless pGL4 vector was arbitrarily set as 1 . The values are shown as the mean \pm SD of triplicate assays. kazato et al., 1997; Oguchi and Kimura, 1998). These studies presented different results regarding the region of the TTF-1 gene that showed the highest promoter activity. This discrepancy may partly be due to the use of FRTL- 5 cells, as this cell line frequently exhibits clonal heterogeneity (Jeker et al., 1999). Our study agrees with a previous report that used transgenic mice, showing that regulatory elements that are essential for TTF-1 expression in thyroid cells exist in genomic regions outside of the TTF-1 5'-flanking region (Pan et al., 2004).

Before drawing this conclusion, further studies are needed. First, even though we confirmed the abundant expression of TTF- 1 mRNA in TCO-1 and $8505 \mathrm{C}$ cells, the expression levels of TTF-1 were much lower than those seen in normal thyroid tissues. This may be due to the fact that TTF-1 expressing thyroid cell lines contain a considerable number of TTF-1 negative cells (Zito et al., 2009). We therefore need to clarify whether such cells may have affected our results. Second, we sequenced the TTF-1 5'flanking region in both TTF-1 positive and negative cell lines and confirmed that there were no mutations or deletions. However, besides mutation analysis, the possibility of methylation of the TTF-1 promoter should be investigated because it can cause epigenetic silencing (Kondo et al., 2009).

In thyroid cell lines we observed higher transcriptional activity in cells containing plasmids that code for a sequence proximal to the transcription start site of exon 1 . Some transcription factors that interact with this region may enhance transcription, whereas factors that interact with the distal region may negatively regulate transcription, although these effects are probably not restricted to TTF-1 positive cells.

Our results showed a striking difference from studies using TTF-1 positive lung cell lines, which showed strong transcriptional activity when the cells were transfected with plasmids similar to ours (Ikeda et al., 1995; Hamdan et al., 1998). A further comparison of the transcriptional activity of the TTF-1 gene between thyroid- and lung-derived cell lines may clarify the factors that negatively regulate TTF- 1 transcription in thyroid cells.

In conclusion, we did not observe differences in promoter activity in a region up to $-2.6 \mathrm{~kb}$ from the proximal transcription start site of the TTF- 1 gene between TTF-1 positive and negative human thyroid cells. These results suggest that the 5'-flanking region of the human TTF -1 gene does not contain sufficient cis-active regulatory information to direct gene expression in human thyroid cells.

\section{Acknowledgments}

This research was supported by the Ministry of Education, Culture, Sports, Science, and Technology of Japan; a Grant-in-Aid for Scientific Research (C, 2008-2010, No. 20590570) and a Research Grant from the Princess Takamatsu Cancer Research Fund (04-23606). 


\section{References}

Hamdan H, Liu H, Li C, Jones C, Lee M, De LR and Minoo P (1998) Structure of the human Nkx2.1 gene. Biochim Biophys Acta 1396:336-348.

Ikeda K, Clark JC, Shaw-White JR, Stahlman MT, Boutell CJ and Whitsett JA (1995) Gene structure and expression of human thyroid transcription factor-1 in respiratory epithelial cells. J Biol Chem 270:8108-8114.

Jeker LT, Hejazi M, Burek CL, Rose NR and Caturegli P (1999) Mouse thyroid culture. Biochem Biophys Res Commun 257:511-515.

Klonisch T, Hoang-Vu C and Hombach-Klonisch S (2009) Thyroid stem cells and cancer. Thyroid 19:1303-1315.

Kondo T, Nakazawa T, Ma D, Niu D, Mochizuki K, Kawasaki T, Nakamura N, Yamane T, Kobayashi M and Katoh R (2009) Epigenetic silencing of TTF-1/NKX2-1 through DNA hypermethylation and histone $\mathrm{H} 3$ modulation in thyroid carcinomas. Lab Invest 89:791-799.

Lazzaro D, Price M, de Felice M and Di Lauro R (1991) The transcription factor TTF-1 is expressed at the onset of thyroid and lung morphogenesis and in restricted regions of the foetal brain. Development 113:1093-1104

Nakazato M, Endo T, Saito T, Harii N and Onaya T (1997) Transcription of the thyroid transcription factor-1 (TTF-1) gene from a newly defined start site: Ositive regulation by TTF-1 in the thyroid. Biochem Biophys Res Commun 238:748752 .

Oguchi H and Kimura S (1998) Multiple transcripts encoded by the thyroid-specific enhancer-binding protein (T/EBP)/thyroid-specific transcription factor-1 (TTF-1) gene: Evidence of autoregulation. Endocrinology 139:1999-2006.
Pan Q, Li C, Xiao J, Kimura S, Rubenstein J, Puelles L and Minoo $\mathrm{P}$ (2004) In vivo characterization of the $N k \times 2.1$ promoter/enhancer elements in transgenic mice. Gene 331:73-82.

Takano T (2007) Fetal cell carcinogenesis of the thyroid: Theory and practice. Semin Cancer Biol 17:233-240.

Takano T, Miyauchi A, Yokozawa T, Matsuzuka F, Maeda I, Kuma K and Amino N (1999) Preoperative diagnosis of thyroid papillary and anaplastic carcinomas by real-time quantitative reverse transcription-polymerase chain reaction of oncofetal fibronectin messenger RNA. Cancer Res 59:4542-4545.

Takano T, Ito Y, Matsuzuka F, Miya A, Kobayashi K, Yoshida H and Miyauchi A (2007) Quantitative measurement of telomerase reverse transcriptase, thyroglobulin and thyroid transcription factor 1 mRNAs in anaplastic thyroid carcinoma tissues and cell lines. Oncol Rep 18:715-720.

Takano T, Asahi S, Matsuzuka F, Hidaka Y, Yoshida H and Miyauchi A (2008) Aspiration biopsy-nucleic acid diagnosis of thyroid malignant lymphoma by vectorette PCR: Experience of eight cases. Leuk Res 32:151-154.

Vliet GV (2003) Development of the thyroid gland: Lessons from congenitally hypothyroid mice and men. Clin Genet 63:445-455.

Yamada H, Takano T, Ito Y, Matsuzuka F, Miya A, Kobayashi K, Yoshida H, Watanabe M, Iwatani Y and Miyauchi A (2009) Expression of nestin mRNA is a differentiation marker in thyroid tumors. Cancer Lett 280:61-64.

Zito G, Richiusa P, Bommarito A, Carissimi E, Russo L, Coppola A, Zerilli M, Rodolico V, Criscimanna A, Amato M, et al. (2009) In vitro identification and characterization of CD133(pos) cancer stem-like cells in anaplastic thyroid carcinoma cell lines. PLoS One 3:e3544.

Associate Editor: Carlos F.M. Menck

License information: This is an open-access article distributed under the terms of the Creative Commons Attribution License, which permits unrestricted use, distribution, and reproduction in any medium, provided the original work is properly cited. 